\title{
Auto-explanation System: Player Satisfaction in Strategy-Based Board Games
}

\author{
Andrew Chiou ${ }^{1}$ and Kok Wai Wong ${ }^{2}$ \\ ${ }^{1}$ School of Computing Sciences, CQUniversity Australia, Rockhampton Campus, \\ Rockhampton Campus, 4702 QLD, Australia \\ a. chiou@cqu .edu. au \\ ${ }^{2}$ School of Information Technology, Murdoch University, \\ Murdoch, Western Australia 6150 \\ k.wong@murdoch.edu.au
}

\begin{abstract}
In computerised version of board games, player satisfaction can be augmented by providing explanation of what the computer based AI analytical processes are. This helps the players to understand the reasoning behind the action taken by the computer AI opponent. This paper proposes a method that provides the mechanism for a game AI to communicate its evaluation processes using descriptive auto-explanation through symbolic reasoning. A case study is presented in an implementation of a game AI opponent that is capable of describing its inferential processes in a tabletop war game.
\end{abstract}

Keywords: board games, player satisfaction, strategy, auto explanation.

\section{Introduction}

The primary purpose of games in any format, be it tabletop board game or its digital counterpart, is to provide entertainment and pleasure to the participants. There are games belonging to the serious game genre. These games, in addition to providing the entertainment and pleasure value, also impart the value of knowledge to the players involved in the current game's subject matter. In the game of chess, it is normal for players to undertake in-depth analysis of completed games. This is to elicit the reasoning behind what and why of specific moves were made during the game. In this way, players augment their pleasure they experience from the games played or being played. This paper will propose a method on how such information can be communicated to the players using its evaluation processes utilising descriptive autoexplanation through symbolic reasoning. This is followed by a case study involving a game $\mathrm{AI}$ in a tabletop strategy-based war game.

\section{Background}

Entertainment computing has becoming a significant area in the field of computing. Recently, it can be observed that there is a shift to focus on the design of the 
entertainment media for individual, so as to increase the perceived value [1]. In the following section, the concept of Player Adaptive Entertainment Computing (PAEC) has been re-examined. The fundamental of the PAEC is to provide personalised experience for each individual when interacting with the entertainment media [2]. The more common area in entertainment computing is digital games. To address the perceived value of players in games in addition to normal game play, this project attempts to implement an auto-explanation mechanism to provide symbolic reasoning to player participants.

\subsection{PAEC Concept}

Perceived value by the users has the power to determine the success of an entertainment media, and thus PAEC is introduced to address this important issue. There are three broad areas of focus in the PAEC: 1 . the player, 2. the content and 3. the entertainment quality. The interaction between the content and player is driven primarily by the value the player perceives. PAEC can be modelled as follow:

$$
\text { perceived } \text { value }_{i}=\frac{\text { experience }+ \text { entertainment quality }}{\text { price }} .
$$

We can see from (1) that the perceived value for player $i$ has several components. The first component, which is experience, refers to the idea that players buy experience and not the particular entertainment products. To the extent, the content of a product enhances the experience and it then increases the player's perceived value. The content that normally enhances experience in entertainment computing includes game resources, platform and the delivery medium. The entertainment quality also increases player's perceived value. Entertainment quality refers to the ways in controlling the mind of the players so that the player feels entertained, which includes inherent interest of events, indirect control techniques, psychological proximity, and player modeling. The way in which a media is produced is often as important as the content itself. Price is also a component of the perceived value. Different components of the player's perceived value provide opportunities for enhancement and management of the interactive content with individual players. From (1), we can see that the perceived value is defined at the individual level (hence the subscript $i$ ). Therefore it is important to identify the components of perceived value that are unique to each player or player base.

\subsection{Auto-explanation}

Previous research has indicated that computerised solutions provided by intelligent systems accompanied by an explanation or justification can make the recommendations more acceptable to end users [3, 4]. In addition, descriptive recommendations using symbolic reasoning or schemas to describe its outcome can facilitate comprehension as compared to numerical-only data. It has been shown that end users require 
data to be charted or graphically presented to facilitate better understanding. The use of symbolic reasoning to visualise qualitative data and information gathering is well established in areas of ethnography [5]. The implementation of specific symbolic types were adapted from and structured around observations such as:

- Space - what are the boundaries of the playing area?

- Actors - what is the purpose of the players, avatars, counters and the playing pieces?

- Activities - what are the [above] activities?

- Events - what specific events is the activity attempting to achieve?

- Time - what are the sequence of events?

\section{Implementation}

The implementation of the auto-explanation mechanism is embedded in the SA $\mathrm{GE}^{3} \mathrm{G}$ GAIA game AI shell prototype [6]. The prototype shell, SA ${ }^{3} \mathrm{GE}$ (Shell for Self-Adapting, Self-Analysing and Self-Aware Game Exponent) was based on specifications of proposed framework in previous work. The purpose of the $\mathrm{SA}^{3} \mathrm{GE}$ production engine is to generate an $\mathrm{AI}$ opponent specific for geomorphic tabletop games and its digital variant. The resulting middleware is GAIA (Game AI Avatar). In Fig. 1, the application provides sockets where the core rules and knowledgebase components can be explicitly instantiated. The core rules are the set of rules specific to the game to function as a filter to ensure that the game play strategies operate within the valid [rule] boundaries of the game. In this experiment, the AI opponent, GAIA is then deployed as a standalone game AI entity where it is fielded against expert human participants to assess its performance in a real-life competitive environment.

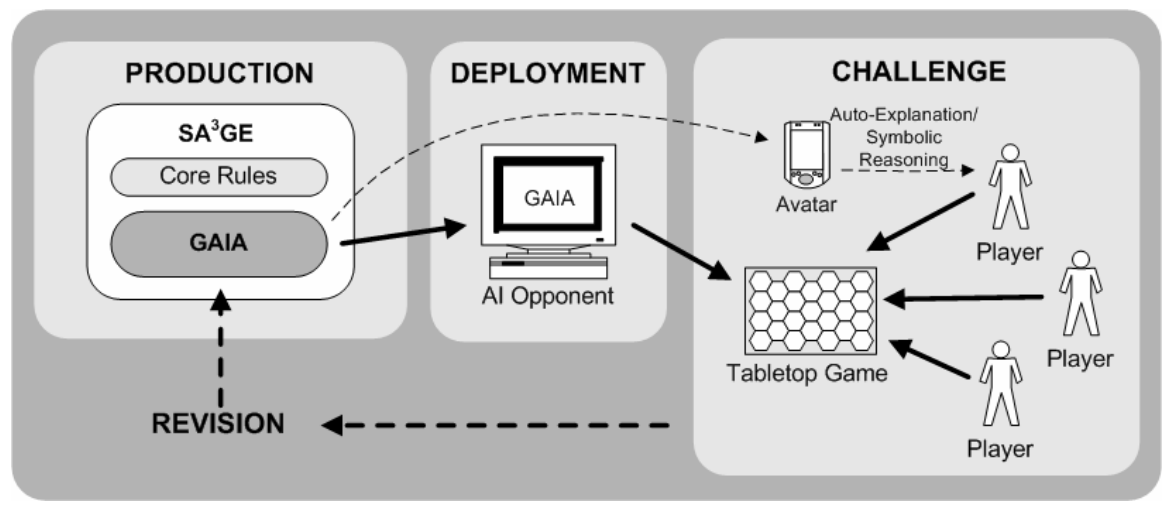

Fig. 1. The $\mathrm{SA}^{3}$ GE-GAIA AI shell with embedded auto-explanation to provide player participants information on specific moves or action considered by the computerised AI playing the role of the avatar 


\section{Auto-explanation Using Symbols}

Descriptive schemas are generated as a series of symbols. There are three types of symbols utilised by GAIA application in providing meaningful advice and suggestions to game players. These are type $S, T$ and $A$. Their technical implementation has been presented in [7]. Each respectively represents deployment strategy, confidence threshold and directed action. The symbols and its purpose will be explained in the following section in the case study. In this way, complex advice such as, "Infantry units can be deployed surrounding, but not within the objective area", can be conveniently expressed. In contrast, a similar output using conventional numerically based data would have been complex and cumbersome.

\section{Case Study}

Tide of Iron is a strategy-based war game played on a hex tiled game board (Fig. 2) [8]. It is a turn-based game for two players. The objective of the game is for players to take the role of either an Axis or Allies military force in order to accomplish specific goals (e.g. secure area of conflict, destroy enemy forces, etc.). The hex-based playing board is geomorphic, allowing the same playing platform to be rearranged to allow different historical battlefield scenarios to be recreated. Different types of resources are available to both players. These are infantry, armour and artillery units. By judicious deployment of these units, players will attempt to defeat the opposition (or score the most points) with minimal lost to its own units.

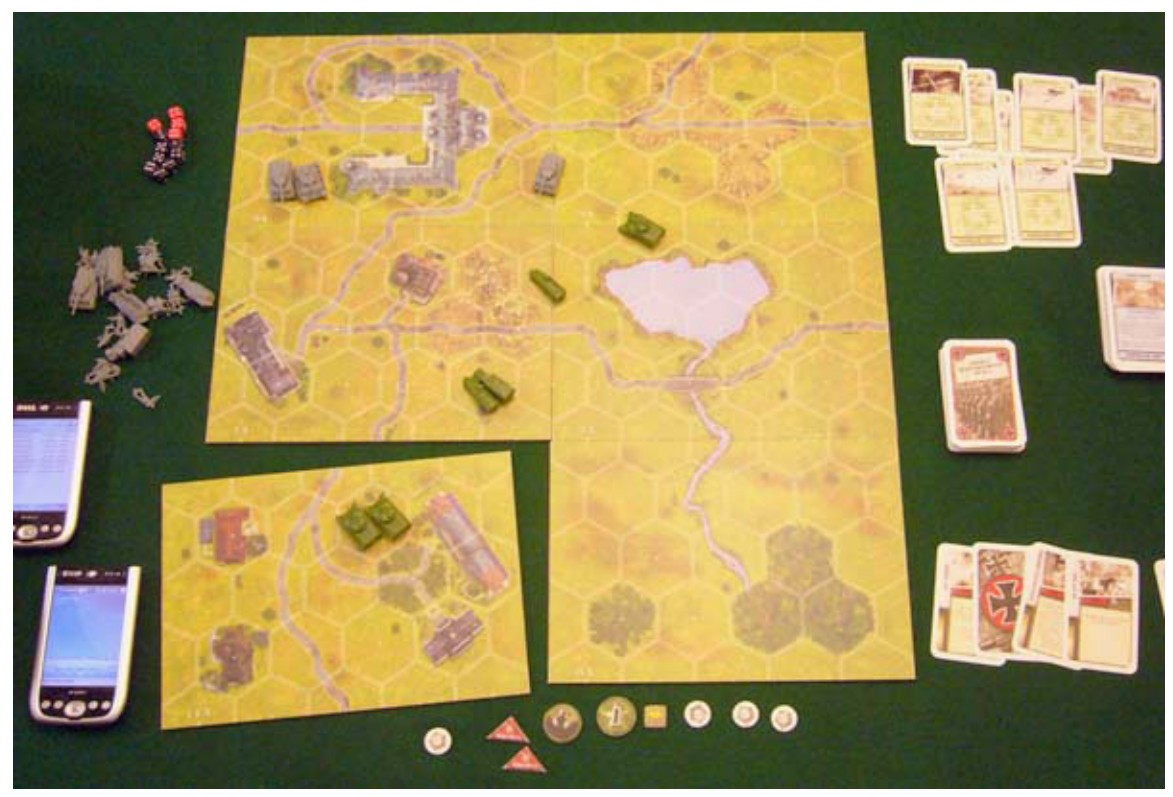

Fig. 2. Physical layout of Tide of Iron [8]. Game board is based on geomorphic arrangement depending on battle scenario. Auto-explanation is generated by GAIA, translated into a script file and transmitted to hand-held devices or netbooks via a web server. 
In the scenario specifically created for the experiment, the objective of the Allied forces (human player) is to capture and hold the town square (in upper left hand corner in Fig. 3). The town is under the control of Axis forces (GAIA). Armour and infantry of the Axis forces surround the objective. The Allied forces should commence by inserting paratroopers in the clear zone (indicated by the three hexes in the lower left). For the Allied forces to gain victory in the confrontation, it has to control at least two hexes occupied by the town square after eight rounds of action. The Axis forces wins immediately if the Allied forces are unable control the required hexes after eight rounds. However, if the Allied forces are able to overrun the enemies after eight rounds but are unable to occupy the required hexes (i.e. the enemies retreat, leaving the town hexes unoccupied by neither forces), this is considered a minor win for the Allied forces.

\subsection{Deployment Strategy, Type $S$}

Deployment strategy refers to the type of possible action to be taken by the player controlling $\mathrm{X}$ force (the opposition is $\mathrm{Y}$ force). A selection of the symbols utilised to represent the strategies is explained in Fig. 3. Depending on the type of board game, the different number of possible strategies and variations appropriate to the type of

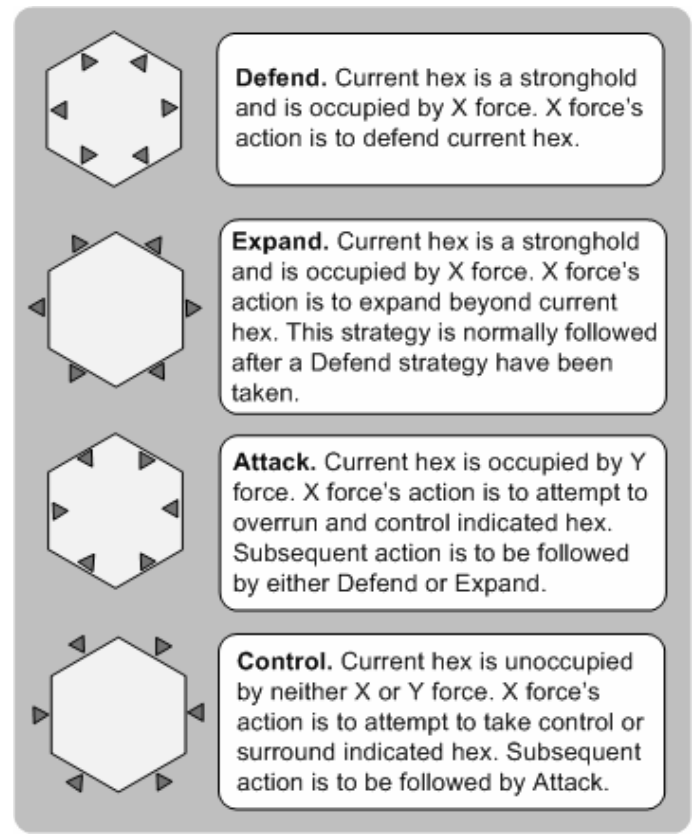

Fig. 3. Legend for the different types of symbols used by GAIA to convey assessment of current game play meaningfully to human participants. There are nine strategies used in Tide of Iron. The empty spaces in the centre of each hex have been reserved for future development. 
game play can range from 4 to 24 . In Tide of Iron, nine strategies are utilised with two variations for each main strategy. After the fifth round (game play normally ends after the ninth round), GAIA generated the auto-explanation in the example output in Fig. 4 representing the lower left zone of the case study scenario. Four critical zones (hexes) were identified where immediate action is required of the human participant playing the role of $\mathrm{X}$ force, where $\mathrm{X}$ is the current player representing the force prepared to move in the current round. $\mathrm{Y}$ force is the opposing player. The critical zones were labelled 1,2,3 and 4, corresponding to the legend in Fig. 5. The auto-explanation in Fig. 5 is in real time, displaying GAIA most recent evaluation of any particular position in the current game play. At present, GAIA is incapable of archiving historical data or keeping track of previous rounds. It is only capable of analysing and providing explanation of a given current position.

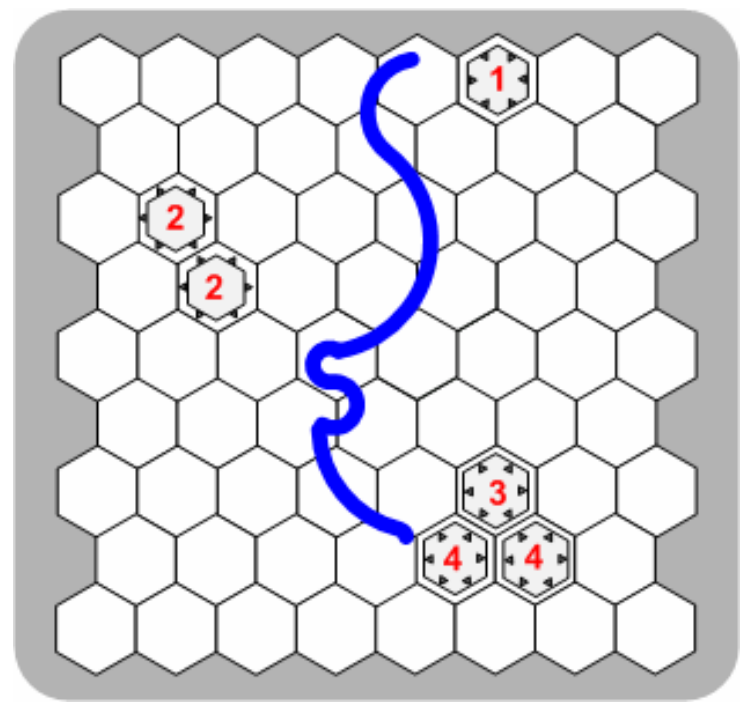

Fig. 4. The critical zone identified by GAIA AI avatar labelled 1, 2, 3 and 4 to correspond with the auto-explanation in Fig. 5. The critical zone are clusters of hexes requiring immediate action in the $n^{\text {th }}$ round during game play. In this case, this is the fifth round.

\subsection{Confidence Threshold, Type T}

In addition, each deployment strategy is complemented by an impact meter to indicate the confidence threshold. This meter provides the confidence level required by players to ensure that the deployment strategy expressed by GAIA is sound or unsound. Darker shade readings indicate that the effectiveness of the deployment strategy is guaranteed. The lighter shade indicates a probable level of effectiveness. The symbolic reasoning for confidence threshold is only meaningful when associated with the relevant deployment strategies inferred previously for each of the critical sectors in Fig. 4 and Fig. 5. 

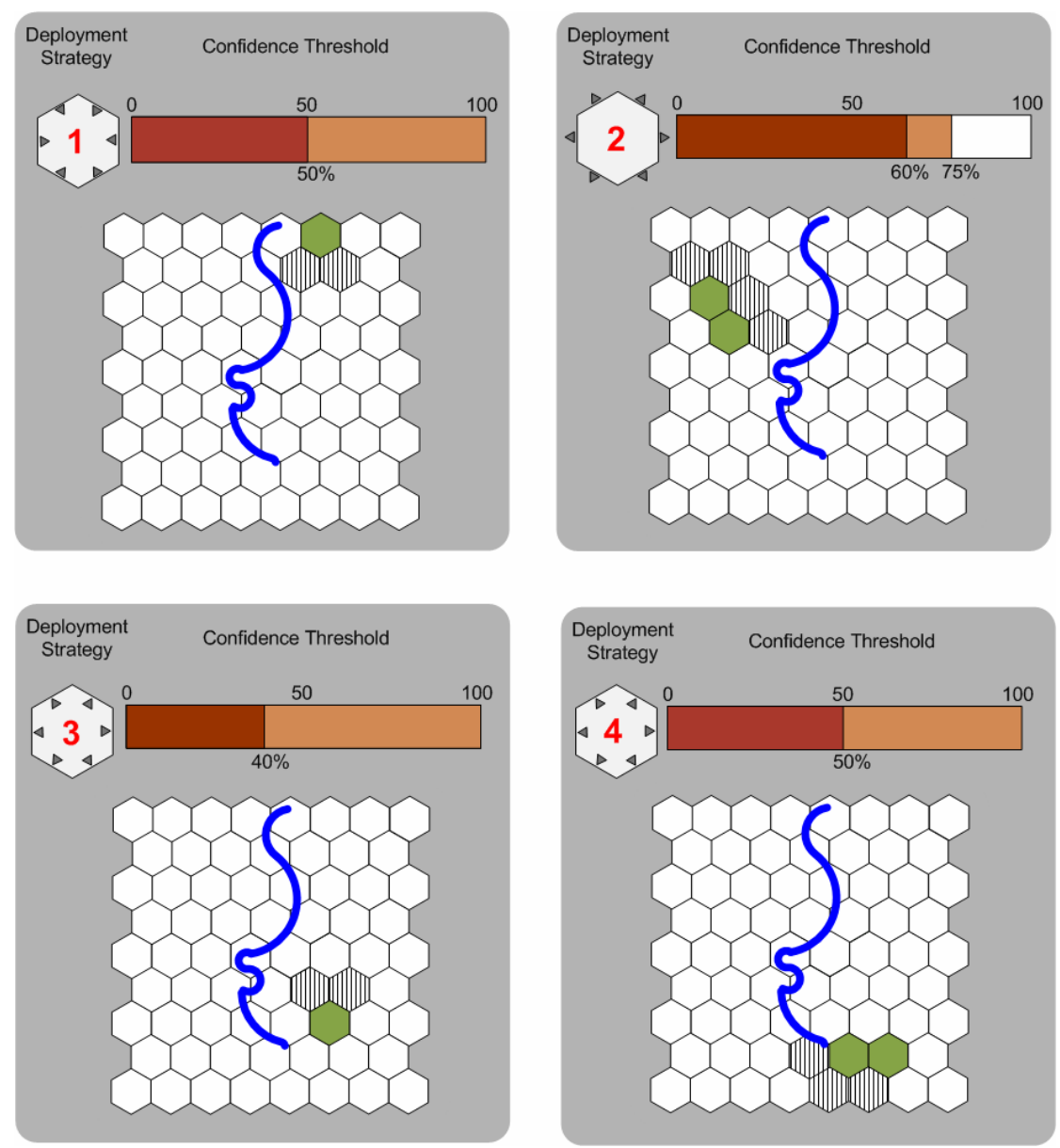

Fig. 5. A stylised and simplified representation of the auto-explanation generated by GAIA to communicate the current analysis for player $\mathrm{X}$ forces against its opponent, $\mathrm{Y}$ forces

\subsection{Directed Action, Type A}

Even though the location have been identified, GAIA requires the proper direction the actions taken should be targeted. An optimal deployment strategy does not necessarily guarantee a high level of success if a less appropriate action has been taken to implement it (e.g. expanding towards an inappropriate hex). Therefore, the application of each action needs to be indicated directionally to maximise the effectiveness of the deployment strategy and its combined actions. To simplify the representation of the recommended actions and to maintain the symbols at a practical and useable level, approximation of the concentration of force along each direction or particular hex corresponding to the critical zones is taken into account. For example, in Fig. 5, the auto-explanation critical zone 3 indicates that expansion be concentrated primarily towards northern hexes with minimal focus on the remaining directions. 


\section{User Satisfaction}

In an initial study involving 22 participants were carried out to provide early indication if auto-explanation generated by a computerised avatar such as GAIA could improve satisfaction. The participants were all male adults in the age range of 16 to 19 . All participants have played the game, Tide of Iron, at least five times in two months prior to the study being carried out. In the study, participants played the game two times over a course of three days. Each game requires an average of four hours to complete. This included the time in consulting the auto-explanation generated by GAIA. After the games were played, the participants were asked to complete a questionnaire. Of the 22 participants, 18 indicated that their level of satisfaction increased with auto-explanation. The remaining 4 indicated that their level of satisfaction remained unchanged or have decreased with auto-explanation. From the 18 that indicated satisfaction, 3 were mildly satisfied, 7 moderated satisfied and 8 highly satisfied as in Fig. 6.

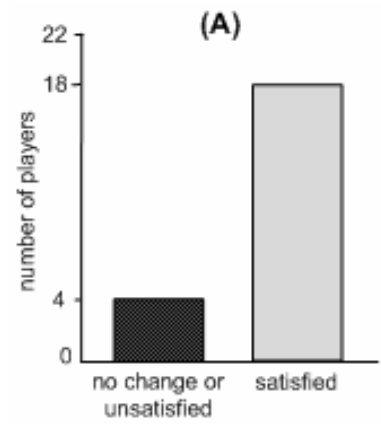

(B)

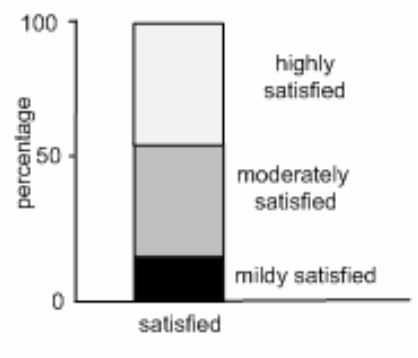

Fig. 6. (A) Participants feedback after playing Tide of Iron with auto-explanation. (B) Composite of participants' degree of satisfaction from those who responded positively.

Therefore, from this early study, it indicates that is feasible for an auto-explanation generated by a computerised avatar such as GAIA, to support and improve a game player's satisfaction during game play. Many factors have yet to be factored and considered. However, this early indication provides the stimulus to allow this proposal to be pursued in more depth.

\section{Summary}

In computerised version of board games, player satisfaction can be augmented by providing explanation of what the computer based AI analytical processes are. This helps the players to understand the reasoning behind the action taken by the computer AI opponent. This paper proposes a method that provides the mechanism for a game AI to communicate its evaluation processes using descriptive auto-explanation through symbolic reasoning. A case study has been presented in an implementation of a game AI opponent that is capable of describing its inferential processes in a tabletop 
war game. In an initial study, early results show that it is feasible that satisfaction of game players provided with auto-explanation can be augmented and improved.

\section{References}

1. Nakatsu, R., Hoshino, J.: Entertainment Computing: Technologies and Applications. Kluwer Academic Publishers, Dordrecht (2002)

2. Nykamp, M.: The Customer Differential: The Complete Guide to Implementing Customer Relationship Management. AMACOM, New York (2001)

3. Gregor, S., Yu, X.: Exploring the Explanatory Capabilities of Intelligent System Technologies. In: Second International Discourse With Fuzzy Logic In The New Millennium. Physica-Verlag, Heidelberg (2000)

4. Gregor, S.: Explanations from Knowledge-based Systems and Cooperative Problem Solving: An Empirical Study. International Journal of Human-Computer Studies 54(1), 81-105 (2001)

5. Crabtree, A.: Designing Collaborative Systems: A Practical Guide to Ethnography. Springer, Berlin (2003)

6. Chiou, A.: A game AI production shell framework: generating AI opponents for geomorphic-isometric strategy games via modeling of expert player intuition. Australian Journal of Intelligent Information Processing Systems 9(4), 50-57 (2008)

7. Chiou, A.: What is it thinking? Game AI opponent computer-human interaction using descriptive schema and explanatory capabilities. In: Proceedings 2nd International Conference on Digital Interactive Media in Entertainment and Arts Perth, Western Australia (2007)

8. http: / /www.boardgamegeek. com/boardgame/22825/tide-of-iron (accessed on: May 20, 2010) 